\title{
Design of novel titanium oxide/nickel oxide multilayer mirror for attosecond soft $\mathrm{x}$ rays
}

\author{
Hiroshi Kumagai and Yusuke Masuda \\ Faculty of Engineering, Osaka City University, 3-3-138 Sugimoto, Sumiyoshiku, Osakashi, Osaka 558-8585, \\ Japan \\ Fax: 81-06-6605-2846, e-mail: kumagai@a-phys.eng.osaka-cu.ac.jp
}

\begin{abstract}
A novel attosecond multilayer mirror was designed at "water-window" wavelengths with a combination of $\mathrm{TiO}$ and $\mathrm{NiO}$, which were the same NaCl-type crystal structures of nearly equal lattice constants $(\mathrm{TiO}=0.41766 \mathrm{~nm}, \mathrm{NiO}=0.41684 \mathrm{~nm})$. The theoretical calculation of the $\mathrm{TiO} / \mathrm{NiO}$ multilayer mirror indicated that the high reflectivity over $50 \%$ was attainable at 2.73 $\mathrm{nm}$ and at an incident angle of $18.9^{\circ}$ from the normal incidence. Moreover, it also indicated that stacking of some multilayer blocks with different periods became effective in reflecting attosecond soft $\mathrm{x}$ rays.
\end{abstract}

Key words: soft $\mathrm{x}$-rays, multilayer, attosecond, titanium oxide, nickel oxide, chirped mirror

\section{INTRODUCTION}

Development of high-performance normal-incidence multilayer optics for the water-window wavelength region between the oxygen and carbon $\mathrm{K}$ absorption edges at 2.33 and $4.36 \mathrm{~nm}$, respectively, where water is relatively transmissive and organic materials are absorptive, has been a technical challenge of great interest. The extremely small periods $\sim 1.2-2.2 \mathrm{~nm}$ of soft-X-ray mirrors require very rigorous specifications to be met with respect to interface roughness and interlayer mixing, because interface roughness on an atomic scale has a substantial effect on soft-x-ray reflectance.

Although the Fresnel coefficients of materials are so small at these wavelengths that a large number of bilayers must be used, which means that the problems of interface roughness and imperfect interfaces due to interlayer mixing become serious, the highest reflectance achieved at water-window wavelengths $(3.11 \mathrm{~nm})$ and near normal incidence $\left(\theta=9.2^{\circ}\right)$ has been reported to be $32 \%$ [1], owing to the various efforts which have been made in this field.

One of the authors have proposed the use of a novel metal oxide multilayer for soft-x-ray mirrors at waterwindow wavelengths [2], because an oxide multilayer can prevent the formation of an alloy at the interface, and the absorption of oxygen in oxides is negligible at the water-window wavelengths; moreover, the metal oxide multilayer can be fabricated by the atomic layer deposition or atomic layer epitaxy (ALE) technique. These techniques can be used to control surfaces on an atomic scale by sequentially dosing the surface with appropriate chemical precursors and then promoting surface chemical reactions which are inherently selflimiting, unlike the conventional fabrication methods of soft-x-ray multilayer mirrors using e-beam evaporation, ion beam sputtering, $\mathrm{rf}$ and $\mathrm{dc}$ magnetron sputtering and so on. We have found that the self-limiting adsorption mechanism works in the fabrication of oxide thin films such as aluminum oxide and titanium oxide[3-6].
One of the authors also demonstrated experimentally high reflectances over $30 \%$ at the water-window wavelengths with 20 bilayers of amorphous titanium oxide and aluminum oxide fabricated by the atomic layer deposition method of controlled growth with sequential surface chemical reactions [7].

Metal oxide multilayers can be fabricated also by ALE technique. With the layer-by-layer growth nature of ALE, the periodicity in multilayer mirrors is able to be controlled with the higher precision [8]. This advantage is also valuable for the fabrication of other functional multilayer structures, such as chirped mirrors, which are useful for compensating for temporal broadening of the x-ray pulse [9].

In this paper, we proposed novel titanium oxide / nickel oxide multilayer mirrors for attosecond soft $x$ rays, and then reported their high reflectances and strong possibility of being attosecond mirrors.

\section{TiO/NiO MIRROR STRUCTURE}

In order to obtain higher reflectivity, a combination of binary materials whose refractive indices are as different as possible and whose absorption coefficients are as small as possible, should be selected. Especially, the titanium L absorption edge, which is located at $2.733 \mathrm{~nm}$, is important to obtain higher reflectivity at the water-window wavelengths. The real part of the Fresnel's coefficient for nickel is relatively high at the wavelength, generating a large difference between the real parts of the binary Fresnel coefficients. Moreover, the imaginary parts of both the Fresnel's coefficients are relatively small. The difference between the real parts of the Fresnel coefficients of the binary oxides is almost the same as that between titanium and nickel. Thus, a combination of $\mathrm{TiO} / \mathrm{NiO}$ becomes a promising candidate for the wavelengths around $2.733 \mathrm{~nm}$. 
In addition, both $\mathrm{TiO}$ and $\mathrm{NiO}$ are the same NaCl-type crystal structures [10]. Therefore, it is possible to make a multilayer of the artificial superlattice between $\mathrm{TiO}$ and $\mathrm{NiO}$ on $\mathrm{MgO}(111)$ substrates, as $\mathrm{MgO}$ also has the NaCl-type crystal structure. Especially, the (111)-oriented reflection planes in the $\mathrm{NaCl}$ lattice model indicates that metal layers and oxygen layers are separate. This separation is suited for ALE methods where the adsorption of vapor precursors should be stopped at one monolayer (ML) automatically through the self-limiting mechanism. Moreover, TiO, NiO, and $\mathrm{MgO}$ have almost the same lattice constants and therefore there is little lattice mismatch between these oxides $\quad(\mathrm{TiO}=0.41766 \mathrm{~nm}, \mathrm{NiO}=$ $0.41684 \mathrm{~nm}, \quad \mathrm{MgO}=0.42112 \mathrm{~nm})$ [10]. Consequently, the combination of $\mathrm{TiO} / \mathrm{NiO}$ has potential enough to be a very attractive waterwindow multilayer mirror.

\section{THEORETICAL CALCULATIONS AND DISCUSSION}

Reflectivities of multilayer mirrors were calculated through the Fresnel's equation with the complex refractive indices. Figure 1 shows the theoretical calculations of the reflectivity (s polarization) of the $(\mathrm{TiO})_{3}(\mathrm{NiO})_{3}$ periodic multilayer mirror, where $\mathrm{x}$ and $\mathrm{y}$ of $(\mathrm{TiO})_{\mathrm{x}}(\mathrm{NiO})_{\mathrm{y}}$ denote layer numbers in one period for $\mathrm{TiO}$ and $\mathrm{NiO}$, respectively. The high reflectivity over $50 \%$ at the wavelength of $2.73 \mathrm{~nm}$ as shown in Fig. 1(a) and an incident angle of $18.9^{\circ}$ from the normal incidence as shown in Fig. 1(b) is obtanable. Fig. 1 (c) shows the theoretical reflectivity as a fuction of numbers of binary layers. With 400 bilayers, $99 \%$ of the highest reflectivity is attainable.

Recently, coherent soft-x-ray source based on the high-order harmonic generation which enables to generate attosecond (as) pulses have received considerable attention. High harmonics generated by the nonlinear interaction of ultrashort pulses from a $800-\mathrm{nm}$ high-intensity Ti:sapphire laser with rare gas is currently the most promising way to generate attosecond pulses. The high harmonic spectrum has the very characteristic shape; it falls off for the first few harmonics, then shows the "plateau" where all the harmonics have the same intensity strength, and finally ends up with the "cutoff" [11]. The cutoff value depends on the IR laser intensity and it is said that the cutoff will extend the water-window region. The ultrashort pulses are the result of the spectral interference of many high-order harmonics. The phase relation between harmonics is not linear but there exists inherent group delay dispersion between harmonics that broadens the pulse duration [12]. Chirped mirrors are one of the solutions proposed to overcome this fundamental limitation. Those mirrors must have both a bandwidth to make interfere as much as possible high-order harmonics and the opposite phase of high-order harmonics that we can control [9]. This induces the particular specification with the multi-block spectral structure based on the multi-periodic
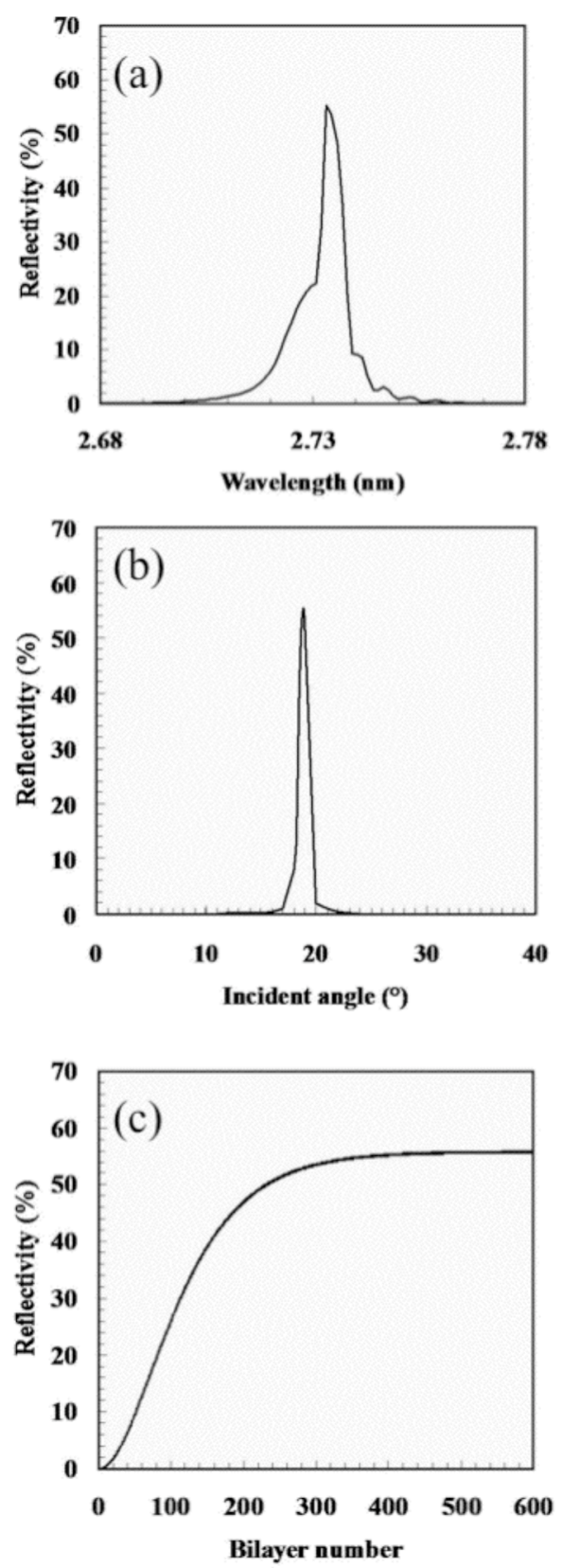

Fig.1: Calculated reflectivities (s-polarization) of the $(\mathrm{TiO})_{3}(\mathrm{NiO})_{3}$-period multilayer mirror as functions of wavelength (a), incident angle (b), and number of bilayers (c).

mirrors. In order to design the attosecond mirror in the water-window wavelengths, we performed the following theoretical calculations.

Figure 2 shows theoretical calculations of the reflectivity of $\left[(\mathrm{TiO})_{3}(\mathrm{NiO})_{3}\right]_{100}$ and $\left[(\mathrm{TiO})_{4}(\mathrm{NiO})_{3}\right]_{100}$ multilayer mirrors in the 2.6-3.3 $\mathrm{nm}$ region and the corresponding single-periodic structures. In Fig. 2, $\left[(\mathrm{TiO})_{3}(\mathrm{NiO})_{3}\right]_{100}=(3,3)_{100}$ represents 100 bilayers of the $(\mathrm{TiO})_{3}(\mathrm{NiO})_{3}$ single-periodic multilayer mirror; $\lambda_{3,3}$ and $\lambda_{4,3}$ indicate the reflection wavelength of $(3,3)_{100}$ and that of $(4,3)_{100}$, respectively. As shown in Fig. 2, 


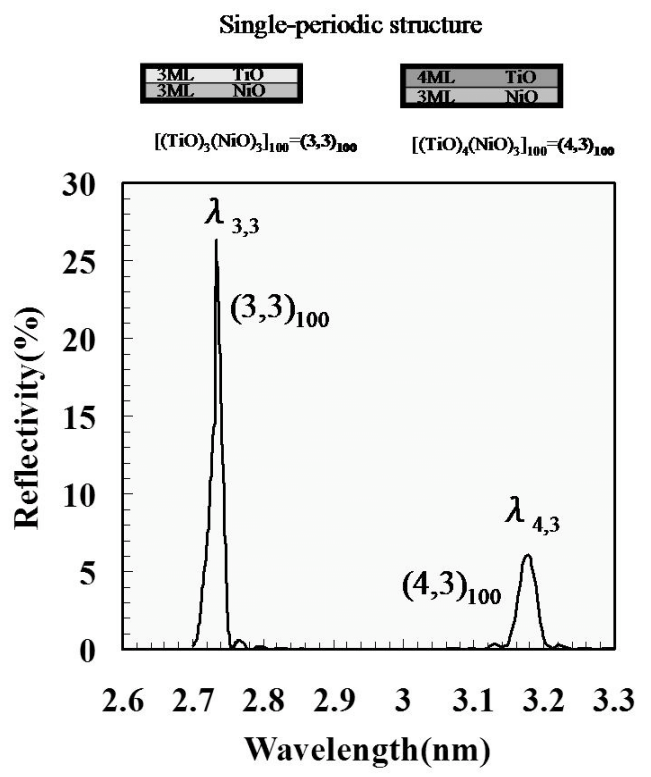

Fig.2: Calculated reflectivities of single-period multilayer mirrors.

each theoretical reflection peak has a narrow bandwidth, and there is a wide gap between neighbors. This property is, indeed, undesirable for a broadband mirror. This disadvantage is caused by the discrete thickness of ML, from the layer-by-layer growth of ALE. However, adoption of the multi-periodic structure can solve the above problem.

In order to control the reflection wavelength precisely, the multi-periodic structure should be proposed [8]. The reflection wavelength of the multi-periodic mirror is determined by the combined ratio of the periodic layers. With the use of this simple combination rule, multiperiodic mirrors at the selected wavelength are designed. Figure 3 indicates two examples of the calculated reflectivity of multi-period mirrors. In Fig. 3, a reflection peak midway of $\lambda_{3,3}$ and $\lambda_{4,3}$, $\left(\lambda_{3,3}+\lambda_{4,3}\right) / 2=2.956 \mathrm{~nm}$ is produced by a combination of $(\mathrm{TiO})_{3}(\mathrm{NiO})_{3}$ and $(\mathrm{TiO})_{4}(\mathrm{NiO})_{3}$ in the ratio of $1: 1$. Correspondingly, $\left(8 \lambda_{3,3}+\lambda_{4,3}\right) / 9$ $=2.780 \mathrm{~nm}$ mirror is obtained from $(\mathrm{TiO})_{3}(\mathrm{NiO})_{3}:(\mathrm{TiO})_{4}(\mathrm{NiO})_{3}=8: 1$. It is important that one period of $(\mathrm{TiO})_{4}(\mathrm{NiO})_{3}$, should be inserted uniformly into the major period, $(\mathrm{TiO})_{3}(\mathrm{NiO})_{3}$ to obtain a higher reflectivity at $2.78 \mathrm{~nm}$. With the use of this method, the wavelength for the reflection peak is controllable fractionally.

Next, a multi-period structure like the so-called "supermirror" is useful for broadband reflection in the water-window. This structure is based on the depth-graded stacking of some blocks which have different wavelengths where reflections peak. Figure 4 shows the calculated reflection peaks of various single-period mirrors with the same number of bilayers in the wavelength region from $2.7 \mathrm{~nm}$ to $2.9 \mathrm{~nm}$. In this figure, each calculated reflection peak is controlled to be near neighbors. The reason that the reflection peaks around 2.733

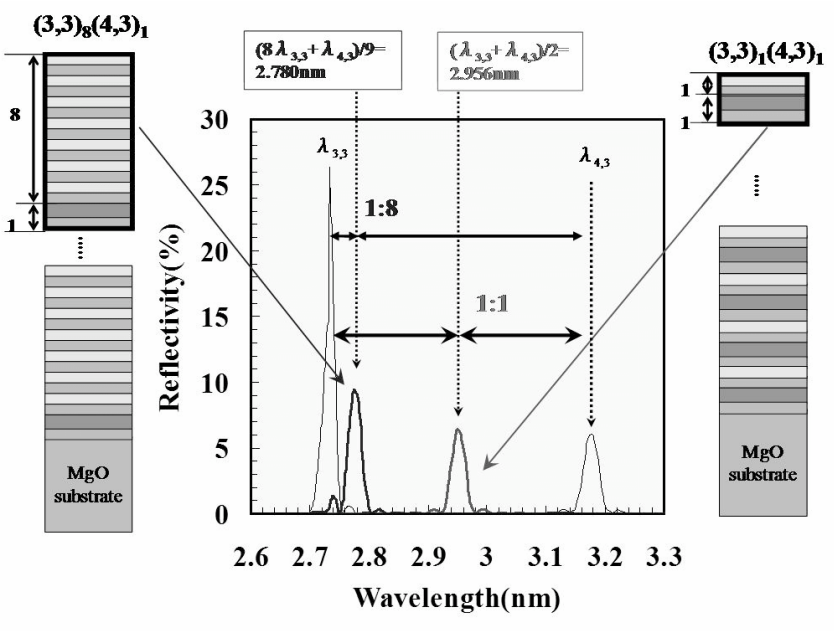

Fig.3: Calculated reflectivities of the multi-period structure mirror.

$\mathrm{nm}$ are outstanding is due to the anomalous dispersion around the titanium $\mathrm{L}$ absorption edge.

Figure 5 shows the results of these theoretical calculations. Fig. 5(a) shows the calculated reflectivity (black line) and the phase relation (gray line) of the chirped mirror. It is note that the chirped mirror can give the reflectivity of about $1-5 \%$ over the large bandwidth covering from $2.73 \mathrm{~nm}$ to $2.89 \mathrm{~nm}$ in the water-window. As shown in Fig. 5 (b), the shortest pulse of 150 as is obtainable theoretically when it can interfer with each other over the spectral range in this mirror in phase. By varying the number of blocks and the thickness of the multilayers, phase variations and reflectances can be designed for your request.

\begin{tabular}{|c|c|c|c|c|c|c|c|c|}
\hline$\left.\left[\left(\mathrm{TTO}^{2}\right) 3(\mathrm{NiO})\right] \mathrm{s}\right][(\mathrm{TiO}) 4(\mathrm{NiO}) 3] \mathrm{y}=[\mathrm{x}: \mathrm{y}]$ & (3,3) ingle & [19:1] & [10:1] & [8:1] & [6:1] & [i:1] & [3:1] & [2:1] \\
\hline reflection wavelenghth peak (mm) & 2.733 & 2.751 & 2.770 & 2.779 & 2.795 & 2.819 & 2.844 & 2.879 \\
\hline
\end{tabular}

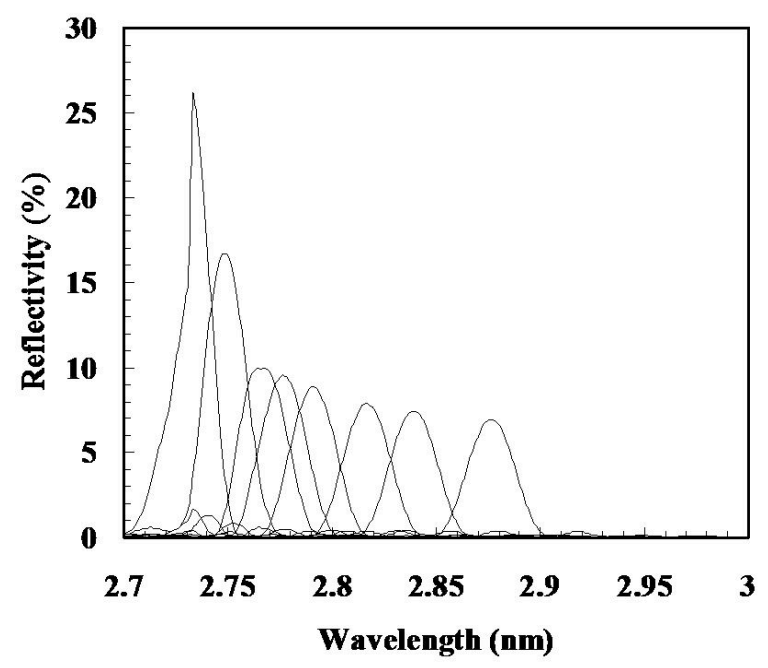

Fig. 4 Calculated reflectivities of various single-period mirrors in the wavelength region from $2.7 \mathrm{~nm}$ to $2.9 \mathrm{~nm}$. 


\section{CONCLUSION}

We have proposed the novel $\mathrm{TiO} / \mathrm{NiO}$ multilayer mirror based on an atomic layer epitaxy technique to fabricate high-reflection robust mirrors for attosecond soft $x$ rays at the water-window wavelengths. The layer thickness of the multilayer mirror is controllable exactly by ALE of controlled growth with sequential surface chemical reactions. This is advantagous for the artificial superlattice multilayer of $\mathrm{TiO} / \mathrm{NiO}$ on $\mathrm{MgO}(111)$, which has NaCl-type (111)-reflection planes where metal layers or oxygen layers are alternative. The theoretical calculation of the periodic $\mathrm{TiO} / \mathrm{NiO}$ multilayer mirror indicated high reflectance over $50 \%$ at a wavelength of $2.73 \mathrm{~nm}$ and an incident angle of $18.9^{\circ}$ from the normal incidence. Moreover, it also indicated that the multi-block structure could control the reflectivity and the phase of a 150-attosecond pulse.
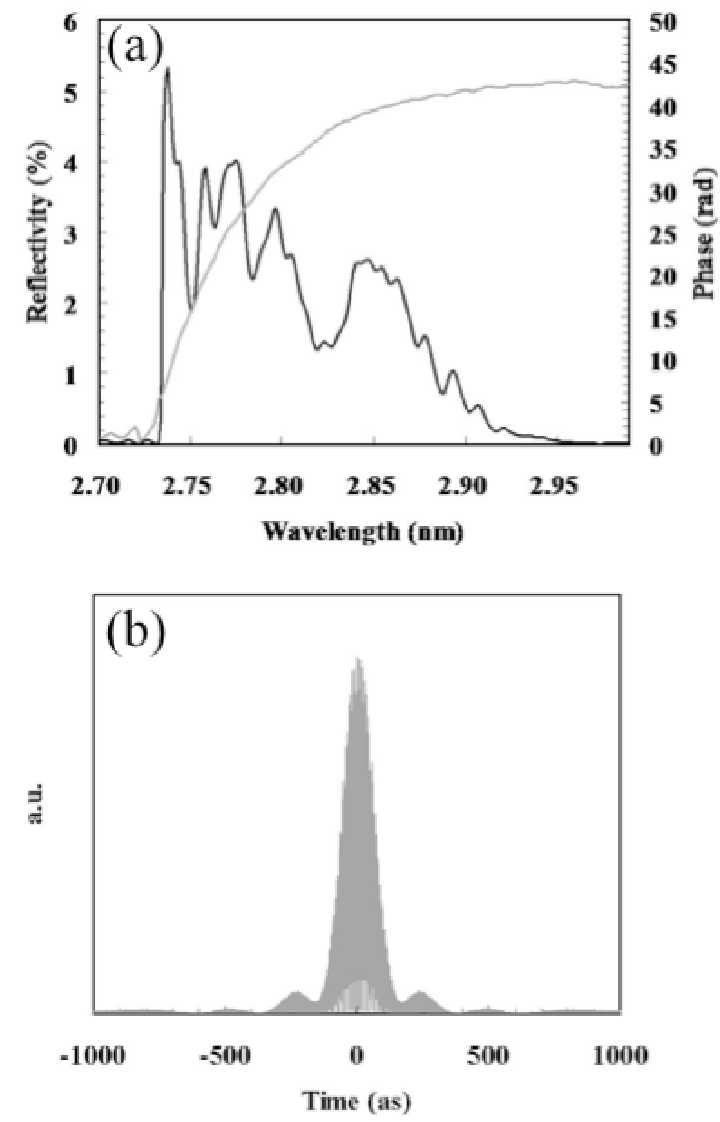

Fig.5: Calculated reflectivity (black line) and phase relation (gray line) of the attosecond mirror (a) and Fourier transformation of the reflectivity (b).

\section{REFERENCES}

[1] F. Salmassi and E. M. Gullikson, Abstracts of the 8th International Conference on the Physics of $X$-ray Multilayer Structures, 39 (2006).

[2] H. Kumagai, M. Matsumoto, Y. Kawamura, K. Toyoda, and M. Obara, Jpn. J. Appl. Phys. 32, 70867089 (1994).

[3] H. Kumagai, K. Toyoda, M. Matsumoto, and M. Obara, Jpn. J. Appl. Phys. 32, 6137-6140 (1993).

[4] H. Kumagai and K. Toyoda, Appl. Surf. Sci. 82/83, 481-486 (1994).

[5] H. Kumagai, M. Matsumoto, K. Toyoda, M. Obara, and M. Suzuki, Thin Solid Films 263, 47-53 (1995).

[6] H. Kumagai, M. Matsumoto, K. Toyoda, and M. Obara, J. Mater. Sci. Lett. 15, 1081-1083 (1996).

[7] H. Kumagai, K. Toyoda, K. Kobayashi, M. Obara, and Y. Iimura, Appl. Phys. Lett., 70, 23382340 (1997).

[8] M. Ishii, S. Iwai, H. Kawata, T. Ueki, and Y. Aoyagi, J. Cryst. Growth. 180, 15-21 (1997)

[9] A.-S. Morlens, M.-F. Ravet, V. Laude, R. López-Martens, M. Roulliay, A. Jérome, F. Delmotte, F. Bridou, S. Kazamias, P. Balcou, and P. Zeitoun, Proc. of SPIE 5963, 59630W (2005)

[10] A. A. Valeeva, A. A. Rempel, W. Sprengela and H.-E. Schaefera, Phys. Chem. Chem. Phys. 5, 2304-2307 (2003).

[11] K. Midorikawa, Springer Handbook of Lasers and Optics, Springer, 819-827 (2007).

[12] Y. Mairesse, A. de Bohan, L. Franzinski, H. Merdji, P. Monchicourt, P. Breger, M. Kovacev, B. Carré, H. Muller, P. Agostini, and P. Saliéres, Science 302, 1540-1543 (2003)

(Received July 14, 2009;Accepted Septembe 17, 2009) 